\title{
Effect of the Dynamic Ergodic Divertor in the TEXTOR Tokamak on MHD Stability, Plasma Rotation and Transport
}

\author{
R. C. Wolf ${ }^{1}$, W. Biel ${ }^{1}$, M. F. M. de Bock², K. H. Finken ${ }^{1}$, S. Günter ${ }^{3}$, G. M. D. Hogeweij ${ }^{2}$,
} M. Jakubowski ${ }^{1}$, R. J. E. Jaspers ${ }^{2}$, A. Krämer-Flecken ${ }^{1}$, H. R. Koslowski ${ }^{1}$, M. Lehnen ${ }^{1}$, Y. Liang $^{1}$, S. K. Varshney ${ }^{2}$, M. von Hellermann ${ }^{2}$, Q. Yu ${ }^{3}$, O. Zimmermann ${ }^{1}$, S. Abdullaev ${ }^{1}$, A. J. H. Donné ${ }^{2}$, G. Matsunaga ${ }^{4}$, S. Ohdachi ${ }^{5}$, U. Samm ${ }^{1}$, B. Schweer ${ }^{1}$, K. Toi $^{5}$, M. Tokar ${ }^{1}$, B. Unterberg ${ }^{1}$, E. Westerhof ${ }^{2}$ and the TEXTOR Team

1) Institut für Plasmaphysik, Forschungszentrum Jülich, Association EURATOM/FZJ, Trilateral Euregio Cluster, D-52425 Jülich, Germany, www.fz-juelich.de

2) FOM-Institute for Plasma Physics Rijnhuizen, Association EURATOM-FOM, Trilateral Euregio Cluster, PO Box 1207, 3430 BE Nieuwegein, The Netherlands, www.rijnh.nl

3) Max-Planck-Institut für Plasmaphysik, Association EURATOM-IPP, Boltzmannstr.2, D-85748 Garching, Germany

4) Japan Atomic Energy Research Institute, Department of Fusion Plasma Research, Naka Fusion Research Establishment, Japan

5) National Institute for Fusion Science, Oroshi-cho, Toki-shi, Gifu 509-5292, Japan e-mail contact of main author: r.wolf@fz-juelich.de

\begin{abstract}
With the Dynamic Ergodic Divertor (DED) in TEXTOR fundamental effects of the coupling of external magnetic field perturbations to the confined plasma have been studied. The non-linear coupling between external and internal modes has been verified experimentally. The critical perturbation field for the excitation of an $m / n=2 / 1$ tearing mode depends not only on the magnitude but also on the direction of the toroidal angular momentum input by neutral beam injection. Below the excitation threshold of this mode a toroidal spin-up of the plasma has been observed, which is independent of the rotation direction of the external perturbation field.
\end{abstract}

\section{Introduction}

The importance of magnetic field stochastization for stability and transport in both tokamaks and stellarators is increasingly recognized. In stellarators the characteristic magnetic island structure is utilized in the island divertor and led to the discovery of the high density H-mode [1]. In tokamaks, indications exist that certain MHD instabilities can be controlled or even suppressed with helical magnetic field perturbations: (A) It has been suggested that the interaction of neoclassical tearing modes (NTMs) with different helicity results in a stabilization of the less unstable one [2] $]^{1}$. Similarly, the application of an external helical magnetic field perturbation should result in the stabilization of the NTM. Indeed, first experiments show an effect, although the simultaneous braking of the plasma rotation was also accompanied by a confinement loss [3]. (B) Another application is the mitigation of edge localized modes (ELMs) [4, 5]. Here, recent experiments show the disappearance of strong low frequency ELMs, while maintaining the H-mode pedestal pressure and thus the plasma confinement [6]. In all these cases a critical question is the interaction of the magnetic field perturbation with the magnetically confined plasma and to which extent the beneficial effect of avoiding certain instabilities is outweighed by possible adverse changes of the plasma confinement. For the latter a particular interest lies in the behavior of the plasma rotation or viscosity under the influence of such helical magnetic field perturbations.

\footnotetext{
${ }^{1}$ While in [2] the ratio of parallel to perpendicular heat conduction has been assumed to be $\chi_{\|} \chi_{\perp}=10^{8}$, a more realistic value of $\chi_{\|} \mid \chi_{\perp}=10^{10}$ leads to a reduction of the stabilizing interaction of the modes with different helicity.
} 
In the TEXTOR tokamak a new system of helical magnetic field coils has been installed. The unique feature of this Dynamic Ergodic Divertor (DED) [7, 8] is the capability to apply rotating magnetic perturbation fields with a frequency of up to $10 \mathrm{kHz}$. The system consists of 16 perturbation coils (four quadruples), plus two additional coils for the compensation of the magnetic field imperfections at the feeder regions of the coils. The coils wind helically around the inner, high field side of the torus (major radius $R=1.75 \mathrm{~m}$, minor radius of the circular plasma cross-section typically $a=0.47 \mathrm{~m}$ ) with a pitch corresponding to the magnetic field lines of the flux surface with a safety factor of $q=3$. Depending on the coil connections, principle mode numbers of the DED are $m / n=12 / 4,6 / 2$ or $3 / 1$. While the $m / n=12 / 4$ mode decays rather quickly, affecting mainly the plasma edge, the perturbation field of the $m / n=3 / 1$ configuration penetrates deeply into the plasma (FIG. 1). Accordingly, the main application of the first is the study of the dynamic ergodic divertor concept [9], while the latter is mainly used to study MHD wave excitation, plasma braking or acceleration [10] and error field amplification. The intermediate $m / n=6 / 2$ configuration has not been used up to now.

(a)

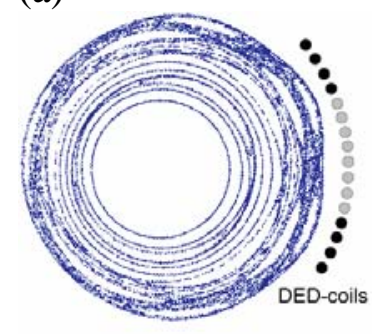

Resonant mode amplitudes for $\mathrm{I}_{\mathrm{OED}}=1.0 \mathrm{kA}$

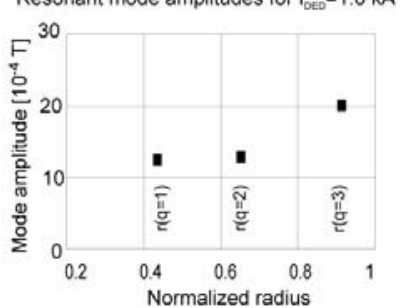

(b)

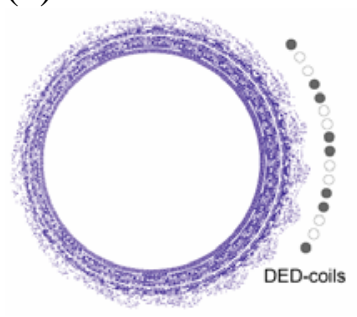

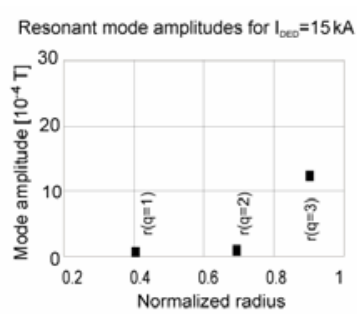

FIG. 1. Poincaré plot of magnetic field topology with (static) DED for (a) the $m / n=3 / 1$ and (b) the 12/4 configuration, calculated from a superposition of the perturbation field (for DED currents, $I_{D E D, 3 / 1}=1.0 \mathrm{kA}$ and $I_{D E D, 12 / 4}=15 \mathrm{kA}$ and a plasma equilibrium with an edge safety factor of $q(a)=4.5$ for the $3 / 1$ and $q(a)=3.3$ for the $12 / 4$ configuration). Also shown are the underlying amplitudes of the magnetic field perturbation for the $m=1,2$ and 3 components at their resonance positions.

This paper focuses on the influence of the DED on the MHD and transport properties of the plasma core, which also means that the emphasis lies on the $m / n=3 / 1$ configuration. For the discussion of the divertor properties of the DED the reader is referred to complementary papers $[9,11]$. First, the interaction of the $m / n=12 / 4$ DED configuration with an intrinsic $m / n=3 / 1$ tearing mode is presented. Subsequently, various results, obtained with the $m / n=3 / 1$ DED configuration, will be discussed. This includes the effect of the DED on transport and plasma rotation at lower perturbation field amplitudes and the controlled excitation of tearing modes at higher amplitudes. The latter capacity has also been used to study the tearing mode avoidance or suppression by electron cyclotron resonance heating (ECRH) and current drive, which is presented in [12].

\section{Interaction between external perturbation field $(m / n=12 / 4)$ and intrinsic tearing modes}

The calculation of the non-linear coupling of NTMs with different helicities predicts a mutual stabilization of the modes, resulting in a survival of the more unstable mode only [2]. Applying the DED in the static $m / n=12 / 4$ configuration, one aspect of this interaction, namely the coupling of the external magnetic field perturbation with an intrinsic $m / n=3 / 1$ 
tearing mode, has been verified. Here however, the interacting magnetic islands, being located at the same rational $q$-surface, have the same helicity.

(a)

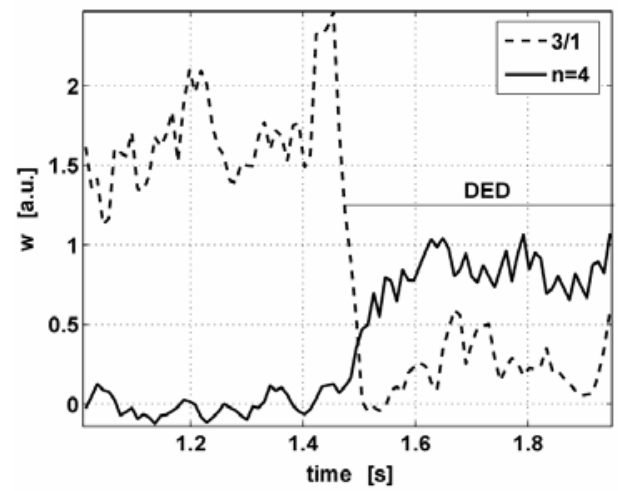

(b)

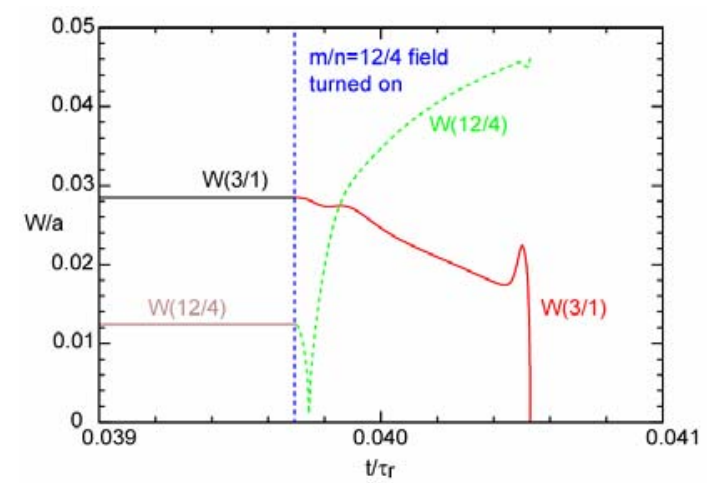

FIG. 2. (a) Measured temporal evolution of the relative change of the island width (assuming $\sqrt{W} \sim$ amplitude of Mirnov signal integrated over the frequency band of the relevant mode) of an $m / n=3 / 1$ and an $n=4$ mode before and during the application of the (static) DED ( $\mathrm{m} / \mathrm{n}=12 / 4$ configuration). When the DED is switched on $\left(I_{D E D}=7 \mathrm{kA}\right)$, the $\mathrm{m} / \mathrm{n}=3 / 1$ mode vanishes. (b) Corresponding temporal evolution ( $\tau_{r}$ : resistive time scale) of the normalized magnetic island width, W/a, as predicted by the numerical calculation of the non-linear coupling between intrinsic mode and external perturbation field [2].

In FIG. 2(a) the temporal evolution of such an intrinsic tearing mode is illustrated. Without DED the plasma shows a weak $m / n=3 / 1$ tearing mode at a frequency of $5.5 \mathrm{kHz}$. At $1.5 \mathrm{~s}$ the DED is switched on, resulting in a stabilization of this mode (in the spectrogram the mode disappears without slowing down, in contrast to cases where modes lock). In agreement with the experiment, the calculation indicates the existence of an $m / n=3 / 1$ tearing mode before the application of the DED. The experimental q-profile underlying the calculation had to be modified slightly to achieve tearing mode instability, which however still lies within the error bars of the measurement. This only shows that the tearing mode is only marginally unstable, which is at least qualitatively in agreement with the very weak experimental $m / n=3 / 1$ signal. In addition, the calculation shows an $m / n=12 / 4$ harmonic of the tearing mode with a smaller island width, which in the experiment is probably too weak to detect. After the DED field is turned on $\left(B_{r, 12 / 4} / B_{\text {tor }}=5.2 \times 10^{-4}\right.$ at $\left.r=a\right)$, the calculation confirms the suppression of the intrinsic $m / n=3 / 1$ mode which is replaced by a growing $m / n=12 / 4$ island, imposed by the external perturbation. Assuming an arbitrarily fast raising time of the DED coil currents, the growth of the $m / n=12 / 4$ mode in the plasma takes about 0.001 resistive time scales. In the calculation, the stabilization of the $m / n=3 / 1$ mode by the applied $m / n=12 / 4$ helical field is attributed to the change of the local current density profile at the $q=3$ surface by the $m / n=$ $12 / 4$ island and the non-linear interaction between the $m / n=3 / 1$ and 12/4 components.

\section{Effect of external perturbation field $(m / n=3 / 1)$ on transport and plasma rotation at low perturbation amplitude}

The $m / n=3 / 1$ helical magnetic field of the DED penetrates deeply into the plasma and thus strongly influences the transport and stability properties of the core plasma [13]. The general course of the experiments, discussed here, is outlined in FIG. 3. After discharge initiation first neutral beam injection (NBI) is applied. NBI is required for the measurement of the toroidal 
plasma rotation by charge exchange recombination spectroscopy (CXRS) and can constitute a significant source of angular toroidal momentum. As TEXTOR is equipped with two NBI systems, oriented in opposite directions (co and counter with respect to the plasma current), the momentum not only depends on the power level, but also on the balance between the contributions from the two beam lines. Once the plasma has reached stationary conditions with respect to plasma current, heating power, electron and ion temperature, and electron density, the DED coil currents are slowly ramped up to a preprogrammed level. This level is kept constant for about half a second, after which the DED is turned off again.

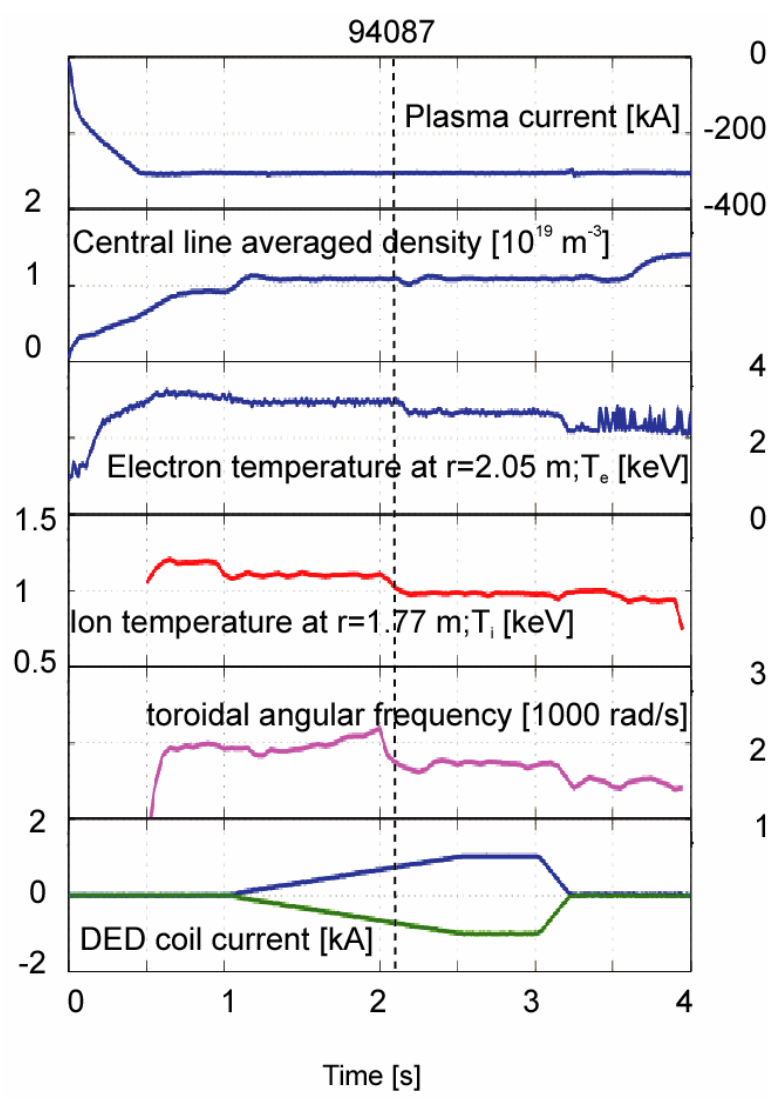

FIG. 3. Time traces of discharge parameters where static DED fields with $m / n=3 / 1$ have been applied. The DED coil currents are slowly ramped up (bottom trace), until an $\mathrm{m} / \mathrm{n}=2 / 1$ tearing mode is induced, indicated by the vertical line.

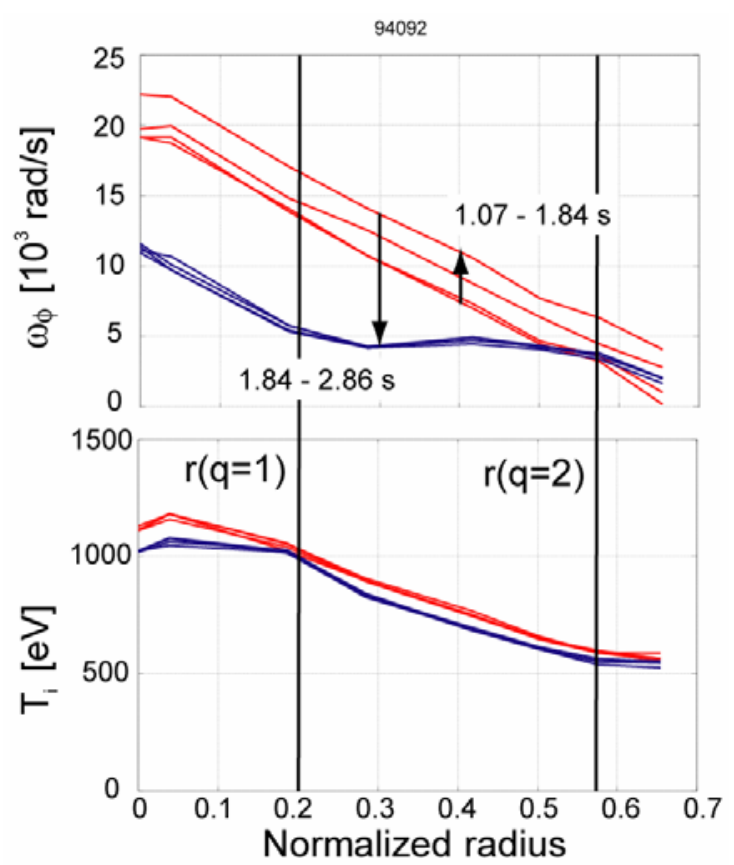

FIG. 4. Temporal evolution of the radial profiles of toroidal rotation and ion temperature with static DED. Before the onset of the tearing mode, the angular frequency increases over the whole profile. The onset of the tearing mode reduces the central temperature peaking and flattens $\omega_{\phi}$ between the $q=1$ and 2 surfaces.

The influence on the toroidal plasma rotation has been studied for different combinations of perturbation field rotation direction and frequency: Static perturbation without rotation, $1 \mathrm{kHz}$ in direction of the plasma current (co-rotation) and with the same frequency opposite to the plasma current (counter-rotation). FIG. 3 shows the time evolution of characteristic parameters with static DED and low NBI power (0.3 MW, corresponding to about the Ohmic heating power). During the application of the perturbation field two phases can be distinguished. First, at low perturbation amplitude, temperature and density profiles do not change. Remarkably the toroidal angular frequency rises by about $20 \%$ with regard to the rotation produced by NBI alone. The second phase is characterized by the formation of an $m / n=2 / 1$ tearing mode, once the current per DED coil exceeds a critical value of about $0.8 \mathrm{kA}$, which corresponds to a magnetic field perturbation of $1.0 \times 10^{-3} \mathrm{~T}$ at the $q=2$ surface. 
This phase is accompanied by significant reductions of temperatures and rotation frequency. The electron density shows only a small dip at the onset of the tearing mode and recovers immediately, caused by the feedback control of the density. Set to a level of $2 \times 10^{19} \mathrm{~m}^{-3}$, the loss of particle confinement is compensated by a stronger gas fuelling which keeps the density constant. The observed dip is a consequence of the finite response time of the feedback control system. Integrating over the profiles of temperatures and densities, the corresponding loss of thermal energy amounts to $12 \%$. The details of the tearing mode, in particular its excitation properties, are discussed in the next chapter.

In figure FIG. 4 the temporal profile evolution of both ion plasma rotation and temperature is shown. Owing to technical restrictions, the CXRS measurement covers only the inner two thirds of the plasma, ranging from the center to the $q=2$ surface. During the first phase, when the DED coil currents start increasing, $\omega_{\phi}=v_{\phi} / R$ (where $v_{\phi}$ is the Doppler velocity obtained from CXRS) rises over the whole profile, while the ion temperature does not change. At the onset of the tearing mode $\omega_{\phi}$ drops in the plasma center by almost a factor of two, in contrast to the vicinity of the $q=2$ surface, where it further increases. This results in a flat rotation profile between the $q=1$ and 2 surfaces, as if in this region the plasma moved like a rigid body. The ion temperature does not show such a pronounced change, which is also reflected in the quite moderate drop of plasma energy.

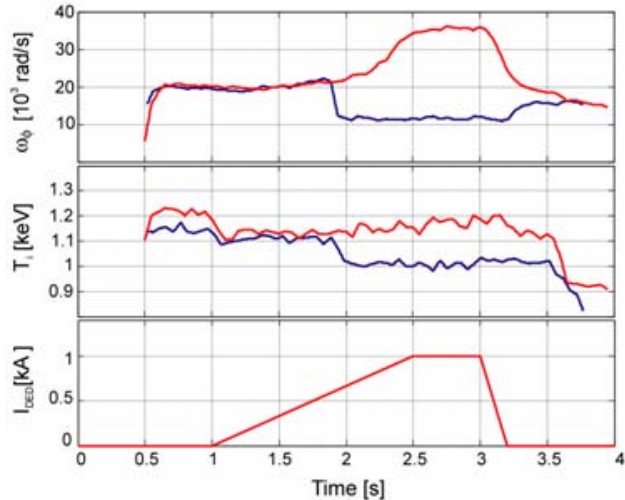

FIG. 5. Temporal evolution of the central toroidal angular frequency and ion temperature at co- (red) and counterrotating (blue) DED. The DED current amplitude, which is the same in both cases, is indicated by the trace at the bottom of the graph.

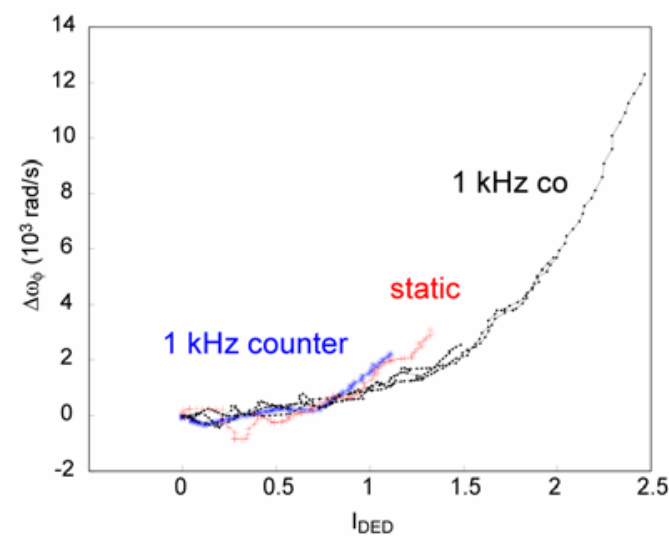

FIG. 6. Dependence of the change of the central toroidal angular frequency on DED current, $I_{D E D}$, for discharges with co-, counter-rotating and static DED, and $0.3 \mathrm{MW}$ co-NBI, before the onset of the $m / n=2 / 1$ tearing mode.

With rotating DED field the temporal evolution of the experiment is basically the same as the one illustrated in figure FIG. 3. The only difference is that the DED coils are now supplied with AC currents to produce a rotating magnetic field perturbation. Rotating the DED with a frequency of $1 \mathrm{kHz}$ opposite to the plasma current (counter-rotation) and NBI, the behavior of the plasma is essentially the same as in the static case (FIG. 5). Consistent with the observations in the static case, the core plasma rotation does not completely brake or lock to the mode. Instead a finite co-rotation is preserved $\left(f=\omega_{\phi}(2 \pi) \approx 1 \mathrm{kHz}\right)$, while the DED perturbation fields are rotating with about the same frequency in the opposite direction.

The most prominent effect is seen when applying a co-rotating DED field. A tearing mode is not excited, although the effective DED current is clearly above the threshold required to 
excite a tearing mode in the static or counter-rotating cases. Instead, as illustrated in FIG. 5, the toroidal rotation continues to rise with the DED current to almost twice the initial level. The relation between central toroidal rotation and DED current amplitude for cases with co-, counter-rotating and static DED is summarized in FIG. 6. Basically the three cases show the same dependence. Only the level to which $I_{D E D}$ can be increased without inducing a tearing mode differs. Interpreting the strength of the DED current as the degree of ergodization [14], the strong dependence of $\omega_{\phi}$ on the DED current is supporting evidence that the magnetic field ergodization is the cause for the strong toroidal spin-up of the plasma.

A tentative explanation could be an enhanced radial transport of the electrons in the open field lines of the ergodic boundary together with the ambipolarity constraint for the ions. This leads to a charge separation which is counteracted by the buildup of a radial electric field, $E_{r}$. The direction of the observed increase of $v_{\phi}$ is consistent with such a mechanism, assuming that the $v_{\phi} \times B_{\theta}$ component in the force balance (with $B_{\theta}$ the poloidal magnetic field) is the dominant term of the change of $E_{r}$ (for probe measurements of the radial electric field at the plasma edge see [9]).

The evidence for changes of the thermal transport before the tearing mode sets in is not entirely conclusive. While the above explanation requires an ergodization at the plasma boundary, consistent with the constant increase of the toroidal angular frequency profile inside the $q=2$ surface, also the question arises to which extent the perturbation fields modify the transport in the plasma core. Within the experimental uncertainties, the profiles of temperature and density remain unaffected by the DED until the $m / n=2 / 1$ mode is excited. Transient impurity transport experiments even suggest a weak increase of the radial transport in both plasma edge and core regions with increasing DED current. Contrary to these observations, heat pulse modulation experiments with ECRH indicate a reduction of the electron heat transport for those plasmas with the largest increase of toroidal angular frequency.

(a)

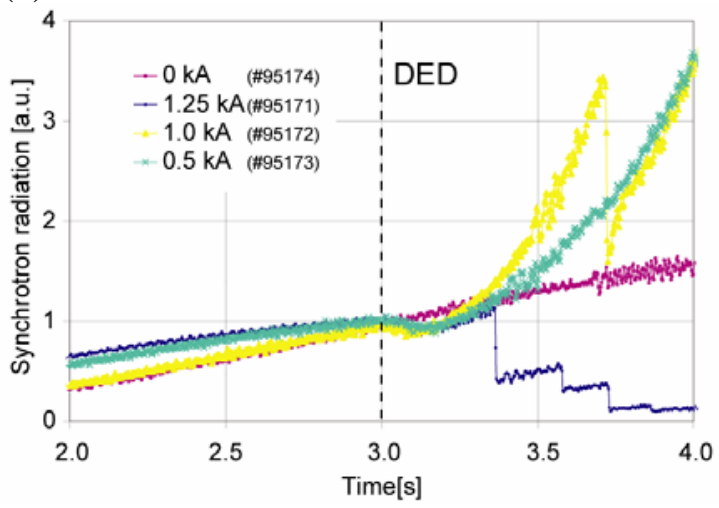

(b)

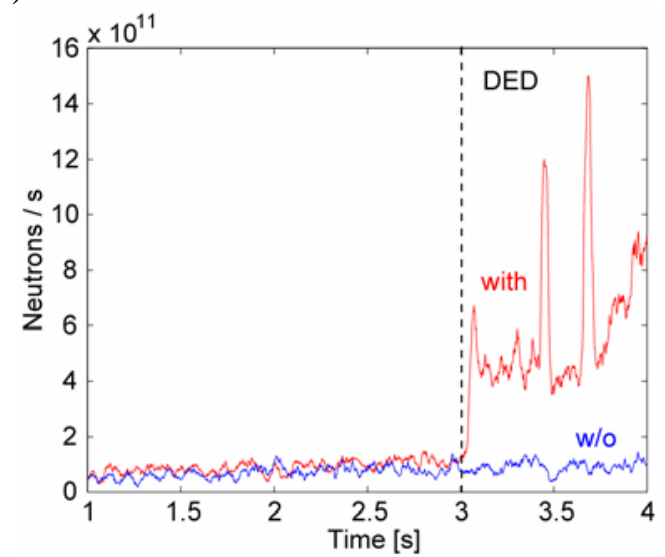

FIG. 7. (a) Synchrotron radiation generated by run-away electrons, comparing discharges without DED and with different levels of DED currents. The DED is switched on at $3.0 \mathrm{~s}$ $\left(I_{D E D}=0.5 \mathrm{kA}\right)$. (b) The loss of fast electrons also results in a strong neutron signal, produced by the impact of the fast electrons on wall components. The sharp drops in (a), corresponding to the burst in (b), indicate some kind of instability, which yet has to be identified.

The existence of an additional loss mechanism by the ergodization is corroborated by experiments where at low densities run-away electrons have been generated and subsequently 
exposed to static DED fields. FIG. 7 clearly shows that the runaway population (measured by the synchrotron emission in the infra-red) is depleted immediately after the DED is turned on and before the tearing mode is excited.

\section{Effect of external perturbation field $(m / n=3 / 1)$ on MHD stability at large perturbation amplitude}

Evidence for the formation of an $m / n=2 / 1$ tearing mode above a critical threshold of the DED amplitude is the local flattening of temperature and density profiles in the vicinity of the $q=2$ surface (FIG. 8) [15]. The helicity is deduced from the relative phase evolution of Mirnov coil signals, which are taken at different poloidal and toroidal positions. Once excited, the mode locks to the external magnetic field perturbation, i.e. for a static DED it does not move with respect to the plasma vessel. After the DED is switched off, the mode persists, but starts to rotate. In some cases, the rotation of the tearing mode does not start immediately after the switch-off of the DED, but is delayed until also the NBI is turned off [16]. To obtain a stationary saturated mode an edge safety factor $q(a) \geq 4.5$ is required. At lower $q(a)$ the plasma tends to disrupt. Instrumental for the formation of the tearing mode is the strong $m / n=2 / 1$ side band of the $3 / 1$ perturbation field. In addition, the tearing mode stability parameter, $\Delta^{\prime}$, is closer to instability at the $q=2$ surface than at $q=3$. Nevertheless, at sufficiently large DED currents (typically $>2 \mathrm{kA}$, compared to $\leq 1 \mathrm{kA}$ for the $2 / 1$ mode) an additional $m / n=3 / 1$ tearing mode can be excited.

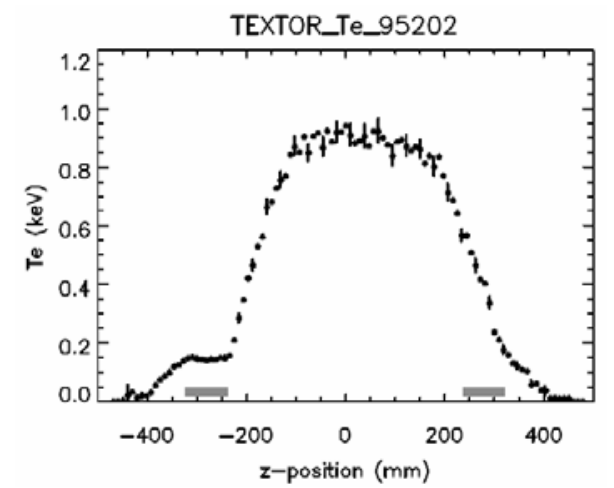

FIG. 8. Electron temperature profile from Thomson scattering [15], clearly showing a shoulder from the magnetic island of the locked $\mathrm{m} / \mathrm{n}=2 / 1$ mode. The $\mathrm{z}$-coordinate roughly corresponds to the minor radius of the plasma. Since, however, the line of sight is shifted by $\Delta r / a \approx 0.2$ away from the plasma center, the profile is asymmetric: For negative $\mathrm{z}$ it goes through the $\mathrm{O}$-point of the island, whereas for positive $\mathrm{z}$ only the $X$-point is seen.

The excitation of the tearing mode is very reproducible and the critical perturbation threshold varies with plasma parameters, such as density, plasma beta or toroidal plasma rotation [17]. In FIG. 9 the different dependencies are shown.

(a)

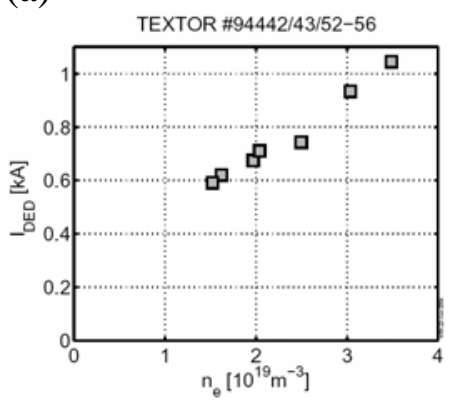

(b)

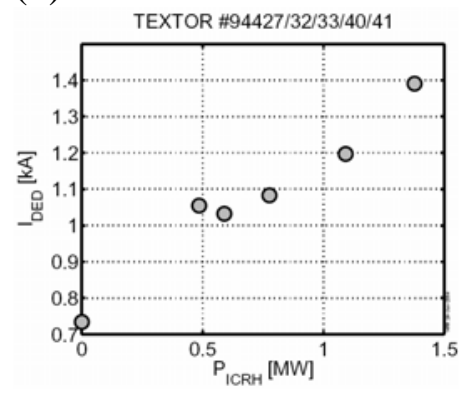

(c)

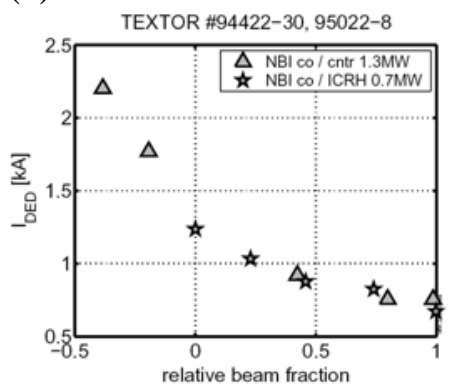

FIG. 9. Dependence of the critical DED current (static operation) to excite a $m=n=2 / 1$ tearing mode on (a) line averaged density, (b) ICRH power (plasma $\beta$ ) and (c) relative beam fraction (angular momentum). In (c) relative beam power fraction corresponds to $\omega_{\phi} \approx-30-$ $+35 \times 10^{3} \mathrm{rad} / \mathrm{s}$. 
The increase of the threshold with density is equivalent to an increase with collisionality, as the heating power was kept constant and thus the temperature decreases. For the power scan ion cyclotron resonance heating (ICRH) was used to avoid a change of the momentum input. Clearly, increasing the heating power, i.e. raising $\beta$, has a stabilizing influence. The most interesting result is shown in FIG. 9(c). Here, the total heating power was kept constant and only the relative neutral beam fraction, defined as $\left(P_{c o}-P_{\text {counter }}\right) /\left(P_{c o}+P_{c o u n t e r}+P_{\text {ICRH }}\right)$ was modified. Also electron density and temperature at the $\mathrm{q}=2$ surface were constant within a few percent. The data clearly show that co-injection in the presence of a static DED perturbation is destabilizing, whereas counter-injection increases the mode threshold. An explanation for this observation is missing up to now. In any case, these results seem inconsistent with recent error field studies on JET, showing a penetration of the perturbation field which does not depend on the NBI direction [18].

\section{Summary and conclusion}

With the DED in TEXTOR important aspects of the interaction of magnetic perturbation fields and the MHD stability and transport properties of the plasma have been studied. The non-linear coupling of the external perturbation $(m / n=12 / 4)$ with internal tearing modes of the same helicity has been confirmed experimentally. The interaction of the DED in the $m / n=3 / 1$ configuration with the plasma shows a challenging dependence of the tearing mode excitation threshold on the toroidal momentum input, which depends on the direction of the NBI. At magnetic perturbation field levels below the threshold for tearing mode excitation a strong toroidal spin up of the plasma in co-current direction is observed, which is independent of the direction of the DED rotation. The observed rotation direction agrees with a radial electric field, produced by an enhanced electron transport in an ergodic edge. The absence of substantial confinement changes suggests that also the plasma viscosity remains unaffected.

This work, supported by the European Communities under the contract of Association between EURATOM-FZJ, FOM and IPP, was carried out within the framework of the European Fusion Programme. The views and opinions expressed herein do not necessarily reflect those of the European Commission.

[1] Mc Cormick et al., Phys. Rev. Lett 89 (2002) 015001

[2] Q. Yu et al., Nucl. Fusion 40 (2000) 2031

[3] La Haye et al., Phys. Plasmas 9 (2002) 2051

[4] H. Tamai et al., Proc. 15th Int. Symp. on Plasma Phys. Control. Fusion, vol. 1, p. 137, IAEA (1995)

[5] A. Grosman et al., J. Nucl. Materials 313-316 (2003) 1314

[6] T. Evans et al., Phys. Rev. Lett. 92 (2004) 235001

[7] K. H. Finken (Guest Editor), Special Issue: Fusion Engineering and Design 37 (1997) 335

[8] K. H. Finken et al., accepted for publication in Plasma Phys. Control Fusion (2004)

[9] B. Unterberg et al., EX/P5-33, this conference

[10] K. H. Finken et al., Nucl. Fusion 99 (2002) 697

[11] M. Lehnen et al., accepted for publication in J. Nucl. Materials (2004)

[12] E. Westerhof et al., EX/P5-16, this conference

[13] K. H. Finken et al., submitted for publication in Phys. Rev. Lett. (2004)

[14] M. C. Jakubowski et al., Nucl. Fusion 44 (2004) 395

[15] S. K. Varshney et al., Proc. $33^{\text {st }}$ EPS Conference on Plasma Phys., London (2004) P1.127

[16] Y. Liang et al., Proc. $31^{\text {st }}$ EPS Conference on Plasma Phys., London (2004) P1.126

[17] H. R. Koslowski et al., Proc. $31^{\text {st }}$ EPS Conference on Plasma Phys., London (2004) P1.124

[18] R. Buttery, private communication (2004) 